\title{
Preconceptual Spectral and Temporal Cues as a Source of Meaning in Speech and Music
}

\author{
Mark Reybrouck $1,2, *\left(\mathbb{D}\right.$ and Piotr Podlipniak ${ }^{3}$ (D) \\ 1 Musicology Research Group, KU Leuven-University of Leuven, 3000 Leuven, Belgium \\ 2 IPEM-Department of Musicology, Ghent University, 9000 Ghent, Belgium \\ 3 Institute of Musicology, Adam Mickiewicz University in Poznań, ul. Umultowska 89D, \\ 61-614 Poznań, Poland; podlip@amu.edu.pl \\ * Correspondence: Mark.Reybrouck@kuleuven.be; Tel.: +32-478-603-479
}

Received: 23 January 2019; Accepted: 26 February 2019; Published: 1 March 2019

\begin{abstract}
This paper explores the importance of preconceptual meaning in speech and music, stressing the role of affective vocalizations as a common ancestral instrument in communicative interactions. Speech and music are sensory rich stimuli, both at the level of production and perception, which involve different body channels, mainly the face and the voice. However, this bimodal approach has been challenged as being too restrictive. A broader conception argues for an action-oriented embodied approach that stresses the reciprocity between multisensory processing and articulatory-motor routines. There is, however, a distinction between language and music, with the latter being largely unable to function referentially. Contrary to the centrifugal tendency of language to direct the attention of the receiver away from the text or speech proper, music is centripetal in directing the listener's attention to the auditory material itself. Sound, therefore, can be considered as the meeting point between speech and music and the question can be raised as to the shared components between the interpretation of sound in the domain of speech and music. In order to answer these questions, this paper elaborates on the following topics: (i) The relationship between speech and music with a special focus on early vocalizations in humans and non-human primates; (ii) the transition from sound to meaning in speech and music; (iii) the role of emotion and affect in early sound processing; (iv) vocalizations and nonverbal affect burst in communicative sound comprehension; and (v) the acoustic features of affective sound with a special emphasis on temporal and spectrographic cues as parts of speech prosody and musical expressiveness.
\end{abstract}

Keywords: preconceptual meaning; affective vocalizations; action-oriented embodied approach; affect burst; speech prosody; musical expressiveness

\section{Introduction}

The problem of meaning extraction in speech and music has received a lot of concern in different fields, such as infant-directed speech and singing, the origins of music perception and cognition, and the primary use of acoustic cues in emotion-driven and affect-laden preverbal communication. This kind of research saw its heyday in the 1990s with major contributions in the field of early music perception [1,2] and preference. Many efforts have been directed towards the study of motherese and infant-directed speech and singing [3-7], the acoustic basis of young children's preference for such kinds of vocal communication [8,9], the musical elements in early affective communication between newborns and caregivers [10-12], and the role of prosodic features in preverbal and early musical communication [13-15]. Most of this research has stressed the extreme sensitivity of young infants for acoustic features of speech and music [16] as well as the existence of early musical predispositions [17-19]. 
Many of these studies-and also some subsequent ones-have emphasized certain commonalities between language and music [19-21], most of them being related to the prosodic and paralinguistic features of language, which can be considered as being musical to some extent. Besides, there are lots of empirical results which indicate that both music and language can prime the meaning of a word and that music meaning is represented in a very similar fashion to language meaning in the human brain $[22,23]$. This observation suggests that propositional semantics that is specific solely to language can be based on broader meaning categories. which are less precise, but not language specific.

Recent developments have provided additional evidence from the domains of comparative $[24,25]$ and evolutionary musicology [26-33], which deal with the evolutionary origins of music by adopting a comparative approach to vocal communication in animals and an evolutionary psychological approach to the emergence of music in the hominin line [34]. These approaches make it possible to tease apart those processes that appear to be innate from those that develop with maturation or acculturation [35]. The animal research provides insights in the role of acoustic cues in nonverbal and preverbal communication [36,37], which are related to affective speech and which can be considered emotional antecedents of music and language [15]. Some of these findings seem to corroborate, to some extent, Darwin's hypothesis on musical protolanguage, which stated that speech and music originated from a common precursor that developed from "the imitation and modification of various natural sounds, the voices of other animals, and man's own instinctive cries" [38] (p. 3). Such a primitive system would have been especially useful in the expression of emotion and music, as we know it nowadays, and should be a behavioral remnant of this early system of communication (see also [39-41]). It is a hypothesis which has been elaborated and restated by modern researchers under the umbrella of the musical protolanguage hypothesis $[20,24,40,42,43]$.

\section{Meaning Before Language and Music}

Meaning can be considered as the result of the interpretation of stimuli by the nervous system. Such interpretation is often described in terms of internal mental representations that animals have of the things, events, and situations in their environment, and it is evolutionary older than their corresponding expressions in language [44] (pp. 4-6). From a phylogenetic perspective, there are three major kinds of meaning which have evolved over time: Meaning as a way to orient oneself in the environment [45], emotions and emotional communication as integral decision mechanisms [46] and as motivational states which are meaningful for survival by providing also a primordial way of interpretation of the external world [47], and referential meaning as the outcome of the appearance of a conceptual mind [48]. Meaning, moreover, can be considered as a basis for communication, using sound as the main source of information as is the case in primate and human vocalizations $[49,50]$. The latter, however, should not be identified solely with speech and singing, which can be contrasted clearly with nonverbal utterances, such as laughter, crying, and mourning. As such, there is a hierarchy in the kind of meaning that is conveyed by sound: There is, first, a distinction between the digital (speech and music) and analog (prosody, laughter, etc.) usage of sound [51]; there is, second, a hierarchical distinction between preconceptual and conceptual meaning with a first level of simple spectral and temporal cues as a source of reflexes and a second level of expressive dynamics and emotional communication [51,52]; there is, third, a level of meaning that is conveyed by means of syntax, such as, e.g., tonality in music and grammatical correctness in speech [53]; and finally, there is a fourth level of propositional semantics and associative meaning [48], such as language's lexicons and, most probably, chimpanzees' referential grunts [54].

Speech is closely related to vocal production and can be studied from a broader stance, including the articulatory, linguistic, and information-conveying point of view. The articulatory approach describes lexical units in terms of gestures that are characterizations of discrete, physical events that unfold during the speech production process [55]. They can be considered basic units of articulatory action, allowing phonology to be described as a set of relations among "phonological events" [56]. These basic units of articulatory routines are discrete gestures, which emerge pre-linguistically as early and gross 
versions of their adult use [55], calling forth the linguistic level of speech processing with articulatory routines that gradually develop into higher-level phonological units that can be contrasted with each other [57]. Linguistic meaning, however, is discrete-digital rather than analog-continuous. It relies on propositional knowledge without direct coupling with the speech signals_as sounding and thus sensory phenomena-and combines referential meaning with particular sound patterns that function as vehicles to convey symbolic meaning. Such a "vehicle mode" of meaning involves referential meaning, which is a representational mode of conveying information, as against the "acoustic mode", which refers merely to the local modulations in sound that are involved in expressive communication of meanings [58].

Speech, as opposed to language as a system, is articulated in real time. As such, it is a sensory rich stimulus. It provides information across multiple modalities, combining both the auditory and visual modalities, as exemplified most typically in the facial expression of audio-visual emotional speech. The latter, together with prosody, cannot be reduced to the control of voice qualities alone, but is closely related to the integration of sensory modalities-with facial and vocal expressions reinforcing each other [13] - and even with the movements of body [59]. Much research on emotional speech (see e.g., [60]), however, has been oriented rather narrowly to facial expressions since it has been hypothesized over a long period of time that judges are more accurate in inferring distinct emotions from facial expressions than from vocal ones. Acoustic cues, on the other hand, have been considered merely as additional features to facial expression, marking only levels of physiological arousal that are less distinctive than those expressed by the face. This conclusion, however, has proved to be erroneous since previous studies have studied only a limited number of acoustic cues, and the arousal differences within emotion families have also been largely neglected [61]. This has been shown in recent studies that used a comprehensive path model of vocal emotion communication, encompassing encoding, transmission, and decoding processes $[62,63]$ to empirically model data sets on emotional expression and recognition from two different cultures and languages. Results of their extended Brunswikian "lens model" [64]-lens equations, hierarchical regression, and multivariate path analysis-, all reflect the strong evidence from past work on the role of arousal in affective communication that vocal sounds primarily convey the arousal state of the sender. It was stated that the "voice is the privileged modality for the expression and communication of arousal and activation, whereas the face is vastly superior with respect to valence" [62] (p. 24).

Additional evidence comes from studies of infants' reactions to parental communicative signals, which have stressed their outstanding discriminative abilities for timing patterns, pitch, loudness, harmonic interval, and voice quality [65]. It seems, moreover, that newborns are very sensitive also to facial expressions, vocalizations, and hand movements, which they can largely imitate to some extent. Such a kind of communicative musicality, as it has been coined [11,66], shows children's awareness of human communicative signals. It is a faculty which is comprehensive, multimodal, and coherent at birth and in the first months after birth [67]. It stresses the conflation of perceptual and motor aspects in speech recognition and vocal expression, bringing together audio-visual, visual-motor, and audio-motor integration.

Music is related to this preverbal communicative expressivity. It precedes or bypasses verbal communication by stressing the sensory richness of the stimuli. As such, it is directed primarily to itself with meaning being self-referential rather than referring to something else. Contrary to the centrifugal tendency of linguistic meaning, where the attention is directed away from the text proper (centrifugal) to grasp the meaning of what is referred to, music has a centripetal tendency in directing the listener's attention to the auditory material of the sounding music itself $[68,69]$. As such, there seems to be a major distinction between language and music, though there are also some commonalities, which stress a number of shared components. This applies in particular to vocal music and its communicative possibilities.

Music, seen from an evolutionarily point of view, is one of the most ancient forms of human communication, with the human voice being probably the most ancestral instrument in human 
music [70]. It can even be questioned in this regard whether music and speech are different, and if so, to what extent [71]. There are two ways to address this question, either by intraspecies or interspecies comparison. An example of the former is the study of para-musical elements in language and para-lingual elements in music [72], such as the use of lexical tone in tone languages and prosody (para-musical) or the use of Leitmotive in music (para-lingual) [20]. Also, the languages based on musical tone systems, such as drum and whistle languages, can be studied in this context [73]. The interspecies comparison, on the other hand, is still more challenging and embraces a very extensive body of research. It has been hypothesized, for example, that singing could have evolved from loud calls by nonhuman primates, such as the Old-World monkeys and apes, which have been considered to be possible precursors of human singing and music. Gibbons, in particular, use vocalizations that elicit emotional responses from human listeners by using acoustic characteristics, such as loudness, acceleration of note rhythm, a final slow-down in rhythm, sounds consisting of alternated exhalation and inhalation, higher pitch frequencies in the central section of the call, pure tone of notes, and frequent accompaniment with piloerection and locomotor displays [36]. All these elements, however, are also used to a different degree in speech.

As such, there is an ability of communication by means of sounds that touches on an evolutionarily old layer of sound communication, which is older than singing and speech. This level is involved in the development of functional sensitivity to a specific class of sounds in ancestral vertebrates both as an aid in identifying and localizing predators and for capturing prey [74]. It is exemplified most typically in the use of alarm calls, which can be considered as a class of punctuate sounds that tend to be short, with sharp and abrupt signal onset, dramatic frequency and amplitude fluctuations, and a chaotic broadband spectral content. There is also a broad class of vocalizations that has been labeled "squeaks, shrieks, and screams" and which have direct impact on animal perception [75]. Their specific designs make them stand out against background noise so as to make them easy to localize. Moreover, they may provoke immediate orienting reactions by other animals in the direction of the calls, in combination with reflexive movements that prepare for flight [76]. Such generalized startle responses are induced also in very young infants, even in the absence of significant previous experience. They are, in fact, reducible to the operation of low-level brainstem and subcortical processes, which are associated with sound localization, orienting, and autonomic responding $[77,78]$. These vocalizations, however, can be exemplary of an intentional, communicative use of sounds which differ functionally from simple auditory sensations, which are prelinguistic default labels of sound sources [79], such as sensation of loudness and low pitch, as a tag of a big animal.

Such vocalizations by animals are not gratuitous. They are used frequently by youngsters as an opportunity to influence the behavior of older and larger individuals by engaging their attention, arousal, and concomitant behavior, sometimes in a very compelling way [80]. It can be questioned, however, whether primates have a theory of mind or act intentionally to influence others. A tentative answer can be found in comparable research in humans into to the neurocognitive mechanisms (auditory prosodic activations) that allow listeners to read the intentions of speakers from vocal prosodic patterns, and which illustrates their anchoring at the interface between auditory and social cognition, involving the cooperation of distributed auditory prosodic, sociocognitive, and cingulo-opercular brain areas [81].

These attention-capturing sounds in animals are often characterized by loud protracted bouts of harsh and variable vocalizations, which include rapidly varying combinations of loud, noisy screams and piercing high-frequency tonal cries, with dramatic amplitude and frequency modulations, which together are able to increase the arousal state of the mother, including human ones [74,82]. It has been shown, moreover, that screaming is one of the most relevant communication signals in humans for survival. By using a recently developed, neurally informed characterization of sounds (modulation power spectrum) see $[83,84]$, it has been demonstrated that human screams cluster within a rather restricted portion of the acoustic space between about 30 and $150 \mathrm{~Hz}$, which corresponds to the 
perceptual attribute of roughness. This acoustic roughness has been found also to engage subcortical structures, which are critical to the rapid appraisal of danger [85].

The vocal repertoire of most primate species, however, is not limited to these attention-capturing sounds. There is also an additional class of sounds, which are referred to as "sonants and gruffs" and which may be considered as structural opposites of these arousal-increasing sounds [74]. Instead of being unpatterned and chaotic, they are tonal and harmonically rich, with a more diffuse regularly patterned broadband spectral structure. Rather than having direct impact on listener's arousal and affect, they seem to induce a less inherent affective force. Their richly structured spectra, moreover, make them even suited for revealing clear cues to the caller's identity since their individual idiosyncrasies impart individually distinctive voice cues that are associated either with the dynamic action of the vocal folds or with the resonance properties of the vocal tract cavities [86,87]. Chimpanzees, likewise, are able to intentionally use grunts as referential calls and to learn new calls from other individuals [54], which represents most probably an early stage of the evolution of lexical meaning (but see [88]). However, although the monkeys' vocal tract is ready to generate speech sounds [89], language and music seem to necessitate more elaborate neural processing mechanisms and vocal control [46].

\section{Affective Sounds and Vocalizations}

Speech —at least in its most primitive appearance-and music seem to share a common affective substrate. Studying emotional communication by means of speech and music, therefore, can benefit from a thorough investigation of their underlying mechanisms. One field of research that has been particularly fruitful in this regard has been the study of auditory affective processing that was conducted in the context of speech prosody [13]. It has been argued, in fact, that two separate neuroanatomic channels with different phylogenetic histories participate in human acoustic communication to support either nonverbal affective vocalization or articulate speech $[90,91]$. This dual-pathway model of human acoustic communication clearly distinguishes the propositional and emotional contents of spoken language, which rely on channels that are seated in separate brain networks that create different data structures, which are known as analogue versus digital (see below). Both channels, however, must coordinate to some extent, but the functional mechanisms and neuroanatomic pathways underlying their intertwined integration are still not totally clear [92].

Affective prosody, further, is opposed to the discrete coding of speech, which is used in the case of phonemes, words, and those aspects of music that consist of pitches and durations. Its expressive dynamics can be modelled more effectively by continuous variables, as is the case with emotional gestures that are shared not only by all humans, but also by a broader group of animals, including many taxa of mammals and even other vertebrates [51]. The same dynamics of affective prosody-as an evolutionarily old form of communication-are to be found, in fact, in the prosody of human language and in the vocal expressions of different mammalian species, which could mean that its use in human acoustic communication has deep phylogenetic roots that are present in the vocal communication systems of nonhuman animals as well. Consistent structures, in fact, can be seen in acoustic signals that communicate affective states, such as high-pitched, tonal sounds in expressions of submission and fear, and low, loud, broadband sounds in expressions of threats and aggression. Animal signals may thus have direct effects on listeners. They may not simply provide information about the caller, but may effectively manage or manipulate the behavior of listeners [93] (see also [76]). This prehuman origin hypothesis of affective prosody locates its grounding in innate mechanisms, which have a prehuman basis and which are used to discriminate between different emotions, both qualitatively (anger, fear, joy, sadness, boredom, etc.) and quantitatively (affect intensity) [52]. It has been shown, moreover, that there exists a functional dissociation between brain regions that process the quality of acoustically conveyed emotions (orbitofrontal cortex) and those that process the intensity of that emotion (amygdala) [94]. Current research has also revealed a high degree of acoustic flexibility in 
attention-attracting sounds in nonhuman mammalian species, which points in the direction of more complex acoustic signaling and processing mechanisms [95].

As such, it can be argued that the study of the faculties of language and music can benefit from a comparative approach that includes communication and cognition in humans and nonhuman animals alike [46]. The capacity to learn language, in fact, requires multiple, separable mechanisms, which include the ability to produce, perceive, and learn complex signals as well as to interpret and control them. Some of them seem to have figured already in the common ancestors of both humans and animals, some others evolved later. Relying on comparative data from living animals, therefore, may be definitively helpful to address these issues. Acoustic signaling in humans, in this view, may have roots in the vocal production, auditory perception, and cognitive processing capabilities of nonhuman mammals, and the study of affective prosody, as a shared component of human speech, music, and nonverbal acoustic communication, in particular, may shed some light on the evolutionary roots of human speech and music as well as the evolution of meaning itself. It is important, in this regard, to consider also the role of iconicity - the similarity between some aspects of sound to some aspects of meaning-in linking the sound to meaning in language. It should be noted, in fact, that affective prosody is considered a paralinguistic property, which accompanies the semantic meaning arising from the symbolic system of human language. The question of how meaning emerges from symbolic signs, therefore, cannot be fully understood by focusing only on prosodical features of language, which work in parallel to the semantic processing. Here, an iconic relationship between sound and the meaning of words that has traditionally been considered as only a marginal property of language (e.g., onomatopoeia, and to some extent also phonaesthemes, i.e., a phoneme or group of phonemes, which has recognizable semantic associations as the result of appearing in a number of words with similar meanings, such as, e.g., the English onset /sn-/ in snarl, snout, sniff, snuffle), has been assumed to serve as an interface for accomplishing the need to map linguistic form to human experience as a vital part of meaning making. Iconicity, thus, has been shown to play an important role for both phylogenetic language evolution (e.g., [96]) and ontogenetic language development (e.g., [97]). This holds in particular for the correspondences between the sound and meaning of words in the affective domain, termed affective iconicity [98], which have been supported by recent empirical results indicating that the specific sound profile of a word can be attributed to a specific affective state, which, in turn, can contribute to the perception of the affective meaning of that word, such as, e.g., whether it designates something positive/negative or arousing/calming [99]. Importantly, the affectivity in the sound of words in a language has been shown to be processed in similar brain regions that are involved in processing other types of affective sounds, such as emotional vocalization and affective prosody $[100,101]$. In addition, such affective potential in the sound of words is even capable of interacting with higher cognitive processes, such as affective evaluation of the words' meaning [102]. All this suggests that consciously experienced meaning is inferred from a number of cues that reflects a hierarchy of sound processing.

It is possible, further, to conceive of this hierarchy in the processing of sounds, reflecting the evolutionary history of human sound communication from early mammals, showing an extension of the perceivable spectrum of sound frequency related to the evolution of the mammalian ear [103], to primates. Non-human primates and early hominins, for example, are an especially interesting group in which to consider the potential affective influence of vocalizations on listeners. Because of their large brains and their phylogenetic proximity to humans, traditional research has focused mostly on "higher-level" cognitive processes that organize communication in higher primates. Yet, they still can rely on the neurophysiological substrates for affective influence, which are still very broadly conserved. It is likely, therefore, that affective influence is an important part of the vocal signals of non-human primates [74]. As such, it is possible to conceive of hierarchical levels of affective signaling, starting from loud calls and vocalizations of early hominids, over prelinguistic affective processing of sound by neonates to infant-directed speech, affective speech, and even music. The step via onomatopoeia and iconicity, finally, could be added as a last step from affective to referential signaling. 
The loud calls of early hominins are exemplified most typically in a broad class of vocalizations with acoustic features that have direct impact on animal perception, as mentioned already above: Sharp signal onsets, dramatic frequency and amplitude fluctuations, and chaotic spectral structures [104]. Neonates are another interesting group for the study of prelinguistic affective processing of sound. They have been shown to possess complex endowments for perceiving and stimulating parental communicative signals by discriminating timing patterns, pitch, loudness, harmonic interval, and voice quality [65]. They also seem to react to the human voice and display imitations of facial expressions, vocalizations, and hand movements, showing an awareness of human signals that is already comprehensive, multimodal, and coherent at birth [67]. As a result, people, all over the world, have capitalized on this sensitivity by developing infant-directed speech or motherese (see below), which is obviously more simplified than adult speech, and which involves exaggerated prosodic features, such as wider excursions of voice pitch, more variable amplitude, tempo, and delivery, and more varied patterns of word stress [74]. All these features have been the subject of research on auditory affective processing, which has been conducted mainly in the context of speech prosody, which has been coined also the "third element of language" [105]. Vocal emotion perception in speech, further, has been studied by using test materials consisting of speech, spoken with various emotional tones by actors, and nonverbal interjections or affect bursts, such as laughter or screams of fear [106] (see for an overview). These vocal expressions, which usually accompany intense emotional feelings, along with the corresponding facial expressions, are closely related to animal affect vocalizations [107], which can be defined as short, emotional non-speech expressions, which comprise both clear non-speech sounds (e.g., laughter) and interjections with a phonemic structure (e.g., 'Wow'), but which exclude verbal interjections that can occur as a different part of speech (like 'Heaven', 'No', etc.)" [108].

These nonverbal affect bursts have proven to be useful for the study of meaning. They provide an interesting class of affective sounds, which have been collected in validated sets of auditory stimuli-such as the Montreal Affective Voices (MAV) [106] and the "Musical Emotional Burst (MEB) for musical equivalents [109]. Using nonverbal sounds, moreover, presents several advantages over verbal ones: The stimuli do not contain semantic information, there are no linguistic barriers, the expression of emotion is more primitive and closer to the affect expressions of animals or human babies, and they are more similar to the Ekman faces [110] used in the visual modality than emotional speech. As such, they avoid possible interactions between affective and semantic content, they can be used for the study of cross-cultural differences, and they allow better comparisons across modalities, as well as studies of cross-modal emotional integration [106].

Affect bursts, however, are limited in their semantic content, but are able to communicate by sound [51,111]. Being evolutionarily older than singing and speech, they have been considered as their precursors to some extent. Singing is one of the interesting ways of sound expression, which goes beyond the transmission of semantic information. It can be questioned, however, whether every kind of music — as an evolved and cultural product—exploits such pre-existing perceptual sensitivities, which were originally evolved thanks to a variety of auditory functions, such as navigating sonic environments and communication by means of singing. Cultural evolution, in this regard, has led to increasingly complex and cumulative musical developments through processes of sensory exploitation [112].

\section{Calls, Vocalizations, and Human Music: Affectively-Based Sound-Meaning Relationships}

Music has inductive power. It can move listeners emotionally and physically by means of the information-processing mechanisms it engages. The majority of these mechanisms, however, did not evolve as music-specific traits. Some of them are related to the processing of sound that is recognized as being similar to voices, objects that are approaching, or the sounds of animals. As such, this processing seems to involve cognitive processes of attraction and cultural transmission mechanisms that have cumulatively and adaptively shaped an enormous variety of signals for social relationships [112]. Music, in this view, is an inherently social phenomenon, and the same holds true for loud calls of 
nonhuman primates, especially those of the Old-World monkeys, which, most likely, were the substrate from which singing could evolve [36].

This brings us to the question of the origins of language and music and their mutual relationship. It has been hypothesized, e.g., that language seems to be more related to logic and the human mind, whereas music should be grounded in emotion and the human body [113] (see for an overview). This dichotomous approach has been questioned, however, in the sense that language and music could evolve from common roots, a common musical protolanguage [24,42]. Especially, the loud calls in modern apes and music in modern humans seem to be derived from such a common ancestral form. The calls are believed to serve a variety of functions, such as territorial advertisement, inter-group intimidation and spacing, announcing the precise locality of specific individuals, food sources, or danger, and strengthening intra-group cohesion. The most likely function of early hominin music, on the other hand, was to display and reinforce the unity of a social group toward other groups [36]. This is obvious in vocalizing and gesturing together in time, where the ability to act musically underlies and supports human companionship. It seems likely, moreover, that the elements of communicative musicality are necessary for joint human expressiveness to arise and that they underlie all human communication $[11,66]$.

As such, it seems that a major ancestral function of calls, protolanguage, and music may be related to several kinds of signaling, attention capturing, affective influence, and group cohesion rather than conveying propositional knowledge that is related to higher level cognitive processes that are involved in the communication of contemporary humans. This brings us to the role of affective semantics, as the domain that studies semantic constructs that are grounded in the perceptual-affective impacts of sound structure [74]. Empirical grounding for that kind of signaling has been provided by a typical class of primate vocalizations, which are known as referential emotive vocalizations [58] and separation calls [114]. There are, in fact, a number of important affective effects of sounds and vocalizations, such as, e.g., attention capturing mechanisms, which are used also in speech directed to young infants with the function to focus and maintain attention and to modulate arousal by using dramatic frequency variations. As such, there is a whole domain of acoustic signals which goes beyond the lexico-semantic level of communication and which is shared between humans and non-human animals. There are, as such, acoustic attributes of aroused vocalizations which are shared across many mammalian species and which humans can use also to infer emotional content. Humans, as a rule, use multiple acoustic parameters to infer relative arousal in vocalizations, but they mainly rely on the fundamental frequency and spectral centre of gravity to identify higher arousal vocalizations across animal species, thus suggesting the existence of fundamental mechanisms of vocal expressions that are shared among vertebrates, and which could represent a homologous signaling system [115].

Such core affective effects of vocal signals may be functional. Yet they do not undercut the role of cognition and the possibility of more complex communicative processes and outcomes, such as speech communication in people. The latter can be seen as a refinement of phylogenetically older vocal production and perception abilities that are shared with non-human animals [91]. These abilities may scaffold, in part, an increasing communicative complexity, which means that at least some of the semantic complexity of human language might capitalize on affectively-based sound-meaning relationships. It is probable, therefore, that evolutionarily older ways of interpreting acoustical cues can be involved in the construction of more complex meaning. Such preprepared or early acquired sound-sense relationships represent a form of intrinsic or original meaning that provides a natural foundation from which increasingly complex semantic systems may be constructed, both developmentally and evolutionarily. This foundation can explain the universal tendency first observed by Köhler [116] (pp. 224-225) to associate pseudowords, such as takete or kiki, with spiky shapes whereas malumba or bouba are associated with round shapes [117]. It has been shown, moreover, that the communicative importance of the affective influence of vocal signals does not disappear when brains get larger and their potential for cognitive, evaluative control of behavior increases. It is likely, 
therefore, that complex communicative processes exploit and build on the phylogenetically-ancient and widespread affective effects of vocal signals [74] (p. 183).

\section{Sound Communication, Emotion, and Affective Speech}

Sounds can have a considerable affective effect on listeners and this holds true also for non-human animals that use many of their vocal signals precisely to exert these effects. There is, as such, a relationship between the acoustic structure in animal signals and the communicative purposes they purport $[74,112]$. This is obvious in vocalizations of non-human primates, which bear the mark of design for direct effects on the listener's affect and behavior, as exemplified most typically in alarm vocalizations that are produced during encounters with predators [91]. These alarm calls tend to be short, broadband calls, with an abrupt-onset, standing out against background noise, thus being easy to localize. As such, they display acoustic features for capturing and manipulating the attention and arousal in listeners. They have been studied already in the 1970s in the context of agonistic vocalizations that are involved in confrontations or competitions with others. Among their most important features is a low fundamental frequency $\left(\mathrm{F}_{0}\right)$ and a tendency towards aperiodicity, with a possible explanation that low, broadband sounds with a wide frequency range are often tied to body size and hostile intent. Such sounds, presumably, can induce fear in the receivers. High pitched sounds with tone-like high $\mathrm{F}_{0}$, on the contrary, are related to appeasement and are often produced to reduce fear in listeners $[118,119]$. This illustrates again how sound is often more important than semantic meaning in animals' signals.

Similar findings have been reported also for humans. Prohibitive utterances across cultures, for example, contain similar acoustic features, such as a fast rising amplitude, lowered pitch, and small repertoires [112]. A more elaborated field of research, however, is the study of motherese or infant-directed speech [65]. Mothers, as a rule, speak in short bursts and talk in an inviting sing-song manner with the baby occasionally answering back. Young infants, moreover, stimulate their caregivers to a kind of musical or poetic speech, which can move into wordless song with imitative, rhythmic, and repetitive nonsense sounds. Such baby-mother interactions imply communicative interactions, which have also been called "communicative musicality" [11]. They suggest an awareness of human signals which is present at birth, with newborns reacting to the human voice and imitating facial expressions, vocalizations, and hand movements. It means that young infants possess complex endowments for perceiving and stimulating parental communicative signals by discriminating timing patterns, pitch, loudness, harmonic interval, and voice quality [65]. Effective communication, in this view, must be held by means other than lexical meaning, grammar, and syntax, with mothers and babies being highly "attuned" to the vocal and physical gestures of the mother. Both seem to explore pitch-space in a methodical manner over short and long intervals of time [11]. This has been reported extensively by the Papoušeks [6,19], who both have stressed the importance of early childhood musical behaviors as forms of play to nurture children's exploratory competence. They have studied intensively infant-caregiver interactions and focused on the musicality of these interactions, stressing the indivisibility of music and movement. It has been found, in fact, that music and movement share a dynamic structure that supports universal expressions of emotion as exemplified in particular in infants' predispositions for perceptual correspondences between music and movement. This ability, further, seems to be possible by the existence of prototypical emotion-specific dynamic contours, but also by isomorphic structural relationships between music and movement [120].

They found out that the parent's multimodal stimulation is, so to say, tailored to the infant's early competence for perceiving information through different senses and that "regular synchronization of vocal and kinaesthetic patterns provides the infant with multimodal sensory information including tactile, kinaesthetic and visual information." [6] (p. 100). Similar findings have been reported by Trevarthen [121], who has centered on the temporal characteristics of the infant-caregiver interaction. The rhythmicity of this interaction can be described as the capacity of the infant to follow and respond to temporal regularities in vocalization and movement, and to initiate temporally regular sets of 
vocalizations and movements. What he proposes is a conceptual framework to explore the expression and development of communication or intersubjectivity through empirical observations and analyses of infant-caregiver interaction. It enables the sharing of patterned time with others and facilitates harmonizing the affective state and interaction [27].

As such, there seems to be an evolutionarily old layer of sound communication that exists in speech, but that arouses emotion in singing as well. This happens in a hierarchic order with the evolutionarily older elements being most basic and effective, and those which are acquired in processes of socialization being most subtle and conventional. Primitive affective vocalizations, therefore, are considered as more authentic and more truly felt information than conventional and ritual information [10,122], and a great deal of music is also designed specifically to give rise to these affective effects [74].

\section{Sound/Speech Understanding and the Gestural Approach}

Language and music can be considered as sound-signal using communication systems. There is, however, a distinction with respect to their respective semantics, which can be either lexico-semantic or action-oriented. In language, as well as in music, the vocal or acoustic characteristics may help to convey an impression, but it has been shown that the position of the eyebrows and the facial expression as a whole, may have the same function [119]. Many facial gestures, in fact, are part of a multi-modal array of signals, and facial expressions may even influence the acoustic cues of the expression by vocal tract deformation [13].

This brings us to the question of bimodality and audiovisual integration of emotional expressions [123]. Even in visible emotion, for example, the auditory modality can carry strong information, which is not only related to the consequences of the facial gestures [13]. In this context, it is important to remind the musicality of infant-caregiver interactions with synchronous stimulation that provides continuous multimodal sensory information (see above). This multimodal stimulation, further, entails processes of affective and behavioral resonance in the sense that the neurophysiological organization of behavior depends on a reciprocal influence between systems that guides both the production, perception, interpretation, and response to the behavior of others, somewhat reminiscent of the discovery of mirror and canonical neuron systems in primate brains [124]. This means that seeing an object or an action performed by someone else can activate the same neurons as when one is performing this action oneself. However, the multimodal stimulation can be even stronger. It has been shown, for example, that if acoustic speech is the main medium for phonetic decoding, some integration with the visual modus cannot be avoided [125]. As such, there is a lot of interest in the role of the co-occurrence of sight and sound, with a special focus on research on emotion effects on voice and speech [61].

Multimodal stimulation entails interactions between individuals, which is obvious in the ability to vocalize and gesture together-as in synchronous chorusing and gesturing-both in humans and nonhuman primates [126]. The ability to act musically and to move sympathetically with each other, accordingly, seems to be the vehicle for carrying emotions from one to someone else. It underlies human companionship in the sense that elements of communicative musicality are necessary for joint human expressiveness to arise [11].

Speech, as a later evolutionarily development, pays tribute to this interactive, gestural approach. It is a basic claim of articulatory phonology, which states that articulatory gestures and gestural organization can be used to capture both categorical and gradient information [55]. They can be described as events that unfold during speech production and whose consequences can be observed in the movements of the speech articulators. Gestures, in this view, are dynamic articulatory structures, which consist of the formation and release of constrictions in the vocal tract. As such, they can be described in terms of task-dynamics, which have been used to model different kinds of coordinated multi-articulator actions, such as reaching and speaking. It means also that the same gestural structures 
may simultaneously characterize phonological properties of the utterance (contrastive units and syntagmatic organization) and physical properties.

\section{Sound Comprehension in Speech and Music: Spectral and Temporal Cues}

Articulatory gestures are situated at the productive level of vocal communication. There is, however, also the receptive level, which is related to the recognition of acoustic parameters, such as, for example, spectral cues when we discriminate pitch in music [127] and intonation patterns in speech [128]. Sound comprehension, in this view, should be related to the recognition of the acoustic profiles of vocal expression, as exemplified most typically in emotional expression. It has been stated erroneously that the voice might only reflect arousal. Recent research, using a larger number of parameters, has shown that spectro-temporal parameters play a major role in differentiating qualitative differences between emotions [129]. This is obvious, for example, in the vocal repertoire of most primate species with a clear distinction between squeaks, shrieks, and screams, with direct impact on the listener's arousal and affect, and sonants and gruffs, with structured spectra that provide an excellent medium for revealing clear cues to the identity of the caller (see above). These cues, which are highly idiosyncratic, impart distinctive voice cues in the acoustic features of these calls, which are associated with the patterns of dynamic action of the vocal folds or with the resonance properties of the vocal tract cavities [74,87]. Human infants, accordingly show an impressive acoustic sensitivity, which allow them to discriminate timing patterns, pitch, loudness, harmonic interval, and voice quality [11], with many perceptual biases being in place before articulated speech evolved [112]. Importantly, although all these features depend on acoustic parameters, they are in fact auditory phenomena [79]. It means that the discrimination of vocal cues is the interpretation of sound stimuli by the nervous system influenced by genetic (both species specific and shared with other taxa) and environmental (including cultural) factors.

Music as well as speech can be considered as dynamic stimuli with sounds changing continuously across the time of presentation. This means that new sensory information is added serially during sound presentation, with physiological systems that respond to simple changes in the physical stimulus being continuously active. Sounds, moreover, are dynamic and often require an accrual of information over time to be interpreted [130]. The effects of speech and music, therefore, are related in important ways to the information-processing mechanisms they engage. As a result, humans interpret speech and music sounds not only as expressive information, but also as coherent sound structures, which convey the whole pack of information. Even at this level, however, both speech and music structures are auditory phenomena which rely to a different degree on acoustical cues. In the case of phonemes recognition [131] and timbre discrimination in music [132], the most important cues are spectro-temporal. Spectral cues, in contrast, are crucial in the discrimination of intonation patterns in speech and pitch class structure in music [127].

The main difference between speech and music in this regard consists in the role of particular acoustic cues played in the transmission of meaning. While spectro-temporal cues are crucial for the recognition of words, they seem to be less important as far as the music structure is concerned. It means that spectro-temporal cues evolved in humans as a main source of transmitting lexical meaning. In contrast, spectral cues are important for discrete pitch class discrimination in music-one of the main elements of musical structure-which is deprived of lexical meaning. Nonetheless, spectral cues can contribute to the lexical meaning in tone languages where the relative change of pitch influences the interpretation of the word meaning [133]. Even in tone languages, however, lexical meaning is conveyed mainly by the means of spectro-temporal cues. Similarly, temporal cues can be used as an additional source of information which influences lexical meaning in "quantity languages", which are sensitive to the duration of the segments for the assignment of their meaning [134,135]. It has been shown also that spectral and temporal cues contribute to the signaling of the word meaning in non-tonal languages as well [136], with the extent to which these cues are important for the transmission of lexical meaning being dependent on the particular language. 


\section{Conclusions and Perspectives}

In this paper, we described the role of preconceptual spectral and temporal cues in sound communication and in the emergence of meaning in speech and music, stressing the role of affective vocalizations as a common ancestral instrument in communicative interactions. In an attempt to search for shared components between speech and music, we have stressed their commonalities by defining speech and music as sensory rich stimuli. Their experience, moreover, involves different body channels, such as the face and the voice, but this bimodal approach has proven to be too restrictive. It has been argued, therefore, that an action-oriented approach is more likely to describe the reciprocity between multisensory processing and articulatory-motor routines as phonological primitives. As such, a distinction should be made between language and speech, with the latter being more centripetal in directing the attention of the listener to the sounding material itself, whereas language is mainly centrifugal in directing the attention away from the text to function referentially. There are, however, commonalities as well and the shared component between speech and music is not meaning, but sound. Therefore, to describe quite systematically the transition from sound to meaning in speech and music, one must stress the role of emotion and affect in early sound processing, the role of vocalizations and nonverbal affect burst in communicative sound comprehension, and the acoustic features of affective sound with a special emphasis on temporal and spectrographic cues as parts of speech prosody and musical expressiveness.

One of the major findings in this regard was a kind of hierarchy in the type of meaning that is conveyed, with a distinction between analog and digital usage of the sound. Especially, the role of affective prosody seems to be important here. As a typical example of analog processing, it goes beyond a mere discrete coding of speech and music, stressing the wider possibilities of sound-signal communications systems rather than relying merely on semantic content and propositional knowledge. As such, there seems to be a major ancestral function of affect burst, calls, protolanguage, and music which are related to several kinds of signaling, attention capturing, affective influence, and group cohesion. They hold a place in a developmental continuum at the phylogenetic and ontogenetic level.

The view presented thus suggests that meaning in language and music is a complex phenomenon which is composed of hierarchically organized features, which are mostly related to the interpretation of acoustical cues by the nervous system. The bulk of this interpretation, moreover, is processed at an unconscious level. More studies are needed, however, to better understand the role of spectral and temporal cues as sources of information in the complex process of human communication. Inter-species and inter-cultural comparative studies are especially promising in this respect, but equally important are developmental investigations, which together with genetic research can elucidate the interconnection between the environmental and hereditary information in the process of the development of human vocal communication.

Author Contributions: The first draft of this article was written by M.R. The final version was prepared jointly by M.R and P.P.

Funding: This research received no external funding.

Acknowledgments: We thank the anonymous reviewers. Their critical remarks were very helpful in updating our summary of the current available research.

Conflicts of Interest: The authors declare no conflict of interest.

\section{References}

1. Trehub, S.E.; Hannon, E.E. Infant Music Perception: Domain-General or Domain-Specific Mechanisms? Cognition 2006, 100, 73-99. [CrossRef] [PubMed]

2. Zentner, M.R.; Kagan, J. Perception of Music by Infants. Nature 1996, 383, 29. [CrossRef] [PubMed]

3. Cooper, R.P.; Aslin, R.N. Preference for Infant-Directed Speech in the First Month after Birth. Child. Dev. 1990, 61, 1584-1595. [CrossRef] [PubMed] 
4. Fernald, A.; Kuhl, P. Acoustic Determinants of Infant Preference for Motherese Speech. Infant Behav. Dev. 1987, 10, 279-293. [CrossRef]

5. Masataka, N. Preference for Infant-Directed Singing in 2-Day-Old Hearing Infants of Deaf Parents. Dev. Psychol. 1999, 35, 1001-1005. [CrossRef] [PubMed]

6. Papoušek, M. Intuitive Parenting: A Hidden Source of Musical Stimulation in Infancy. In Musical Beginnings Origins and Development of Musical Competence; Deliège, I., Sloboda, J., Eds.; Oxford University Press: Oxford, NY, USA, 1996; pp. 88-112.

7. Werker, J.F.; McLeod, P.J. Infant Preference for Both Male and Female Infant-Directed Talk: A Developmental Study of Attentional and Affective Responsiveness. Can. J. Psychol. Can. Psychol. 1989, 43, 230-246. [CrossRef]

8. Trainor, L.J. Infant Preferences for Infant-Directed versus Noninfant-Directed Playsongs and Lullabies. Infant Behav. Dev. 1996, 19, 83-92. [CrossRef]

9. Trainor, L.J.; Clark, E.D.; Huntley, A.; Adams, B.A. The Acoustic Basis of Preferences for Infant-Directed Singing. Infant Behav. Dev. 1997, 20, 383-396. [CrossRef]

10. Gorzelańczyk, E.J.; Podlipniak, P. Human Singing as a Form of Bio-Communication. Bio-Algorithms Med. Syst. 2011, 7, 79-83.

11. Malloch, S.N. Mothers and Infants and Communicative Musicality. Music. Sci. 1999, 3, 29-57. [CrossRef]

12. Wermke, K.; Mende, W. Musical Elements in Human Infants' Cries: In the Beginning Is the Melody. Music. Sci. 2009, 13, 151-175. [CrossRef]

13. Aubergé, V.; Cathiard, M. Can We Hear the Prosody of Smile? Speech Commun. 2003, 40, 87-97. [CrossRef]

14. Fernald, A.; Mazzie, C. Prosody and Focus in Speech to Infants and Adults. Dev. Psychol. 1991, 27, $209-221$. [CrossRef]

15. Panksepp, J.; Bernatzky, G. Emotional Sounds and the Brain: The Neuro-Affective Foundations of Musical Appreciation. Behav. Process. 2002, 60, 133-155. [CrossRef]

16. Fassbender, C. Infants' Auditory Sensitivity towards Acoustic Parameters of Speech and Music. In Musical Beginnings Origins and Development of Musical Competence; Deliège, I., Sloboda, J., Eds.; Oxford University Press: Oxford, NY, USA, 1996; pp. 56-87. [CrossRef]

17. Trehub, S.E. Musical Predispositions in Infancy: An Update. In The Cognitive Neuroscience of Music; Peretz, I., Zatorre, R.J., Eds.; Oxford University Press: Oxford, NY, USA, 2003; pp. 2-20. [CrossRef]

18. Trehub, S.E.; Schellenberg, E.; Glenn Hill, D.S. The Origins of Music Perception and Cognition: A Developmental Perspective. In The Origins of Music Perception and Cognition: A Developmental Perspective; Deliège, I., Sloboda, J.A., Eds.; Psychology Press/Erlbaum (UK) Taylor \& Francis: Hove, UK, 1997; pp. 103-128.

19. Papoušek, H. Musicality in Infancy Research: Biological and Cultural Origins of Early Musicality. In Musical Beginnings Origins and Development of Musical Competence; Deliège, I., Sloboda, J.A., Eds.; Oxford University Press: Oxford, NY, USA, 1996; pp. 37-55. [CrossRef]

20. Brown, S. The "Musilanguage" Model of Musical Evolution. In The Origins of Music; Wallin, N.L., Merker, B., Brown, S., Eds.; The MIT Press: Cambridge, MA, USA, 2000; pp. 271-300.

21. Fenk-Oczlon, G.; Fenk, A. Some Parallels between Language and Music from a Cognitive and Evolutionary Perspective. Music. Sci. 2009, 13, 201-226. [CrossRef]

22. Koelsch, S.; Kasper, E.; Sammler, D.; Schulze, K.; Gunter, T.; Friederici, A.D. Music, Language and Meaning: Brain Signatures of Semantic Processing. Nat. Neurosci. 2004, 7, 302-307. [CrossRef] [PubMed]

23. Steinbeis, N.; Koelsch, S. Comparing the Processing of Music and Language Meaning Using EEG and FMRI Provides Evidence for Similar and Distinct Neural Representations. PLoS ONE 2008, 3, e2226. [CrossRef]

24. Fitch, W.T. The Biology and Evolution of Music: A Comparative Perspective. Cognition 2006, 100, $173-215$. [CrossRef] [PubMed]

25. Hauser, M.D.; McDermott, J. The Evolution of the Music Faculty: A Comparative Perspective. Nat. Neurosci. 2003, 6, 663-668. [CrossRef] [PubMed]

26. Cross, I. Music, Cognition, Culture, and Evolution. Ann. N. Y. Acad. Sci. 2001, 930, 28-42. [CrossRef] [PubMed]

27. Cross, I. Music, Mind and Evolution. Psychol. Music 2001, 29, 95-102. [CrossRef]

28. Cross, I. The Evolutionary Nature of Musical Meaning. Music. Sci. 2009, 13, 179-200. [CrossRef] 
29. Huron, D. Is Music an Evolutionary Adaptation? In The Cognitive Neuroscience of Music; Peretz, I., Zatorre, R.J., Eds.; Oxford University Press: Oxford, NY, USA, 2003; pp. 57-75. [CrossRef]

30. McDermott, J.; Hauser, M. The Origins of Music: Innateness, Uniqueness, and Evolution. Music Percept. 2005, 23, 29-59. [CrossRef]

31. Reybrouck, M. From Sound to Music: An Evolutionary Approach to Musical Semantics. Biosemiotics 2013, 6, 585-606. [CrossRef]

32. Tolbert, E. Music and Meaning: An Evolutionary Story. Psychol. Music 2001, 29, 84-94. [CrossRef]

33. Wallin, N.L. Biomusicology: Neurophysiological, Neuropsychological, and Evolutionary Perspectives on the Origins and Purposes of Music; Pendragon Press: Stuyvesant, NY, USA, 1991.

34. Brown, S.; Merker, B.; Wallin, N.L. An Introduction to Evolutionary Musicology. In The Origins of Music; Wallin, N.L., Merker, B., Brown, S., Eds.; The MIT Press: Cambridge, MA, USA, 2000; pp. 3-24. [CrossRef]

35. Drake, C.; Bertrand, D. The Quest for Universals in Temporal Processing in Music. In The Cognitive Neuroscience of Music; Peretz, I., Zatorre, R.J., Eds.; Oxford University Press: Oxford, NY, USA, 2003; pp. 21-31. [CrossRef]

36. Geissmann, T. Gibbon Songs and Human Music from an Evolutionary Perspective. In The Origins of Music; Wallin, N.L., Merker, B., Brown, S., Eds.; The MIT Press: Cambridge, MA, USA, 2000; pp. 103-123.

37. Huron, D.B. The Other Semiotic Legacy of Charles Sanders Peirce: Ethology and Music-Related Emotion. In Music, Analysis, Experience. New Perspectives in Musical Semiotics; Meader, C., Reybrouck, M., Eds.; Leuven University Press: Leuven/Louvain, Belgium, 2015; pp. 185-208.

38. Darwin, C. The Descent of Man, and Selection in Relation to Sex, 1st ed.; John Murray: London, UK, 1871.

39. Ma, W.; Thompson, W.F. Human Emotions Track Changes in the Acoustic Environment. Proc. Natl. Acad. Sci. USA 2015, 112, 14563-14568. [CrossRef] [PubMed]

40. Fitch, W.T. On the Biology and Evolution of Music. Music Percept. 2006, 24, 85-88. [CrossRef]

41. Fitch, W.T. Musical Protolanguage: Darwin's Theory of Language Evolution Revisited. In Birdsong, Speech, and Language: Exploring the Evolution of Mind and Brain; Bolhuis, J.J., Everaert, M., Eds.; The MIT Press: Cambridge, MA, USA, 2013; pp. 489-503.

42. Mithen, S.J. The Singing Neanderthals: The Origins of Music, Language, Mind, and Body; Harvard University Press: Cambridge, UK, 2006.

43. Thompson, W.F.; Marin, M.M.; Stewart, L. Reduced Sensitivity to Emotional Prosody in Congenital Amusia Rekindles the Musical Protolanguage Hypothesis. Proc. Natl. Acad. Sci. USA 2012, 109, 19027-19032. [CrossRef] [PubMed]

44. Hurford, J.R. The Origins of Meaning: Language in the Light of Evolution; Oxford University Press: Oxford, UK, 2007.

45. Hauser, M.D. The Evolution of Communication; The MIT Press: Cambridge, MA, USA, 1996.

46. Fitch, W.T.; Zuberbühler, K. Primate Precursors to Human Language: Beyond Discontinuity. In Evolution of Emotional Communication: From Sounds in Nonhuman Mammals to Speech and Music in Man; Altenmüller, E., Schmidt, S., Zimmermann, E., Eds.; Oxford University Press: Oxford, UK, 2013; Volume 16, pp. $27-48$.

47. Panksepp, J. Affective Consciousness: Core Emotional Feelings in Animals and Humans. Conscious. Cogn. 2005, 14, 30-80. [CrossRef] [PubMed]

48. Bickerton, D. Adam's Tongue: How Humans Made Language, How Language Made Humans; Hill and Wang: New York, NY, USA, 2010.

49. Roederer, J.G. On the Concept of Information and Its Role in Nature. Entropy 2003, 5, 3-33. [CrossRef]

50. Hauser, M.D.; Konishi, M. The Design of Animal Communication; The MIT Press: Cambridge, MA, USA, 1999.

51. Merker, B. Is There a Biology of Music? And Why Does It Matter? In Proceedings of the 5th Triennial ESCOM Conference; Kopiez, R., Lehmann, A.C., Wolther, I., Wolf, C., Eds.; Hanover University of Music and Drama: Hanover, Germany, 2003; pp. 402-405.

52. Zimmermann, E.; Leliveld, L.; Schehka, S. Toward the Evolutionary Roots of Affective Prosody in Human Acoustic Communication: A Comparative Approach to Mammalian Voices. In Evolution of Emotional Communication: From Sounds in Nonhuman Mammals to Speech and Music in Man; Altenmüller, E., Schmidt, S., Zimmermann, E., Eds.; Oxford University Press: Oxford, NY, USA, 2013; pp. 116-132.

53. Merker, B. Music: The Missing Humboldt System. Music. Sci. 2002, 6, 3-21. [CrossRef] 
54. Watson, S.K.; Townsend, S.W.; Schel, A.M.; Wilke, C.; Wallace, E.K.; Cheng, L.; West, V.; Slocombe, K.E. Vocal Learning in the Functionally Referential Food Grunts of Chimpanzees. Curr. Biol. 2015, 25, 495-499. [CrossRef] [PubMed]

55. Browman, C.P.; Goldstein, L. Articulatory Phonology: An Overview. Phonetica 1992, 49, 155-180. [CrossRef] [PubMed]

56. Bird, S.; Klein, E. Phonological Events. J. Linguist. 1990, 26, 33-56. [CrossRef]

57. Studdert-Kennedy, M. The Phoneme as a Perceptuomotor Structure. In Cognitive Science Series Language Perception and Production: Relationships Between Listening, Speaking, Reading and Writing; Allport, A., MacKay, D., Prinz, W., Scheerer, E., Eds.; Academic Press: London, UK, 1987; pp. 67-84.

58. Frayer, D.W.; Nicolay, C. Fossil Evidence for the Origin of Speech Sounds. In The Origin of Music; Wallin, N.L., Merker, B., Brown, S., Eds.; MIT Press: Cambridge, MA, USA, 2000; pp. 271-300.

59. Clynes, M. Sentics: The Touch of Emotions; Anchor Press: Garden City, NY, USA, 1977.

60. Arias, P.; Belin, P.; Aucouturier, J.-J. Auditory Smiles Trigger Unconscious Facial Imitation. Curr. Biol. CB 2018, 28, R782-R783. [CrossRef] [PubMed]

61. Scherer, K.R. Vocal Communication of Emotion: A Review of Research Paradigms. Speech Commun. 2003, 40, 227-256. [CrossRef]

62. Bänziger, T.; Hosoya, G.; Scherer, K.R. Path Models of Vocal Emotion Communication. PLoS ONE 2015, 10, e0136675. [CrossRef] [PubMed]

63. Scherer, K.R.; Clark-Polner, E.; Mortillaro, M. In the Eye of the Beholder? Universality and Cultural Specificity in the Expression and Perception of Emotion. Int. J. Psychol. 2011, 46, 401-435. [CrossRef] [PubMed]

64. Scherer, K.R. Personality Inference from Voice Quality: The Loud Voice of Extroversion. Eur. J. Soc. Psychol. 1978, 8, 467-487. [CrossRef]

65. Trehub, S.E.; Unyk, A.M.; Trainor, L.J. Maternal Singing in Cross-Cultural Perspective. Infant Behav. Dev. 1993, 16, 285-295. [CrossRef]

66. Malloch, S.; Trevarthen, C. The Human Nature of Music. Front. Psychol. 2018, 9, 1680. [CrossRef] [PubMed]

67. Nadel, J.; Butterworth, G. Imitation in Infancy; Nadel, J., Butterworth, G., Eds.; Cambridge University Press: Cambridge, NY, USA, 1999.

68. Kyndrup, M. Mediality and Literature: Literature versus Literature. In Why Study Literature; Nielsen, H.S., Kraglund, R., Eds.; Aarhus University Press: Aarhus, Denmark, 2011; pp. 85-96.

69. Wierød, L.M.L. Where to Draw the Line? In Music, Analysis, Experience; Maeder, C., Reybrouck, M., Eds.; New Perspectives in Musical Semiotics; Leuven University Press: Leuven/Louvain, Belgium, 2015; pp. 135-148.

70. Ewens, G. Die Klänge Afrikas: Zeitgenössische Musik von Kairo Bis Kapstadt; Marino-Verlag: München, Germany, 1995.

71. Brandt, A.; Gebrian, M.; Slevc, L.R. Music and Early Language Acquisition. Front. Psychol. 2012, 3, 1-17. [CrossRef] [PubMed]

72. Rubin, D.C. Memory in Oral Traditions: The Cognitive Psychology of Epic, Ballads, and Counting-Out Rhymes; Oxford University Press: Oxford, NY, USA, 1995.

73. Meyer, J. Typology and Acoustic Strategies of Whistled Languages: Phonetic Comparison and Perceptual Cues of Whistled Vowels. J. Int. Phon. Assoc. 2008, 38, 69-94. [CrossRef]

74. Rendall, D.; Owren, M.J. Vocalizations as Tools for Influencing the Affect and Behavior of Others. Handb. Behav. Neurosci. 2010, 19, 177-185. [CrossRef]

75. Gil-da-Costa, R.; Braun, A.; Lopes, M.; Hauser, M.D.; Carson, R.E.; Herscovitch, P.; Martin, A. Toward an Evolutionary Perspective on Conceptual Representation: Species-Specific Calls Activate Visual and Affective Processing Systems in the Macaque. Proc. Natl. Acad. Sci. USA 2004, 101, 17516-17521. [CrossRef] [PubMed]

76. Owings, D.H.; Morton, E.S. Animal Vocal Communication: A New Approach; Cambridge University Press: Cambridge, UK, 1998.

77. Herzog, M.; Hopf, S. Behavioral Responses to Species-Specific Warning Calls in Infant Squirrel Monkeys Reared in Social Isolation. Am. J. Primatol. 1984, 7, 99-106. [CrossRef]

78. Seyfarth, R.M.; Cheney, D.L.; Marler, P. Monkey Responses to Three Different Alarm Calls: Evidence of Predator Classification and Semantic Communication. Science 1980, 210, 801-803. [CrossRef] [PubMed]

79. Huron, D.B. Voice Leading: The Science Behind a Musical Art; The MIT Press: Cambridge, MA, USA, 2016. 
80. Owren, M.J.; Dieter, J.A.; Seyfarth, R.M.; Cheney, D.L. Vocalizations of Rhesus (Macaca Mulatta) and Japanese (M. Fuscata) Macaques Cross-Fostered between Species Show Evidence of Only Limited Modification. Dev. Psychobiol. 1993, 26, 389-406. [CrossRef] [PubMed]

81. Hellbernd, N.; Sammler, D. Neural Bases of Social Communicative Intentions in Speech. Soc. Cogn. Affect. Neurosci. 2018, 13, 604-615. [CrossRef] [PubMed]

82. Owings, D.H.; Zeifman, D. Human Infant Crying as an Animal Communication System: Insights from an Assessment/Management Approach. In Evolution of Communication Systems: A Comparative Approach; Oller, D.K., Griebel, U., Eds.; MIT Press: Cambridge, MA, USA, 2004; pp. 151-170.

83. Chi, T.; Gao, Y.; Guyton, M.C.; Ru, P.; Shamma, S. Spectro-Temporal Modulation Transfer Functions and Speech Intelligibility. J. Acoust. Soc. Am. 1999, 106, 2719-2732. [CrossRef] [PubMed]

84. Theunissen, F.E.; Elie, J.E. Neural Processing of Natural Sounds. Nat. Rev. Neurosci. 2014, 15, 355-366. [CrossRef] [PubMed]

85. Arnal, L.H.; Flinker, A.; Kleinschmidt, A.; Giraud, A.-L.; Poeppel, D. Human Screams Occupy a Privileged Niche in the Communication Soundscape. Curr. Biol. CB 2015, 25, 2051-2056. [CrossRef] [PubMed]

86. Rendall, D.; Owren, M.J.; Rodman, P.S. The Role of Vocal Tract Filtering in Identity Cueing in Rhesus Monkey (Macaca Mulatta) Vocalizations. J. Acoust. Soc. Am. 1998, 103, 602-614. [CrossRef] [PubMed]

87. Ghazanfar, A.A.; Turesson, H.K.; Maier, J.X.; Van Dinther, R.; Patterson, R.D.; Logothetis, N.K. Vocal-Tract Resonances as Indexical Cues in Rhesus Monkeys. Curr. Biol. 2007, 17, 425-430. [CrossRef] [PubMed]

88. Fischer, J.; Price, T. Meaning, Intention, and Inference in Primate Vocal Communication. Neurosci. Biobehav. Rev. 2017, 82, 22-31. [CrossRef] [PubMed]

89. Fitch, W.T.; De Boer, B.; Mathur, N.; Ghazanfar, A.A. Monkey Vocal Tracts Are Speech-Ready. Sci. Adv. 2016, 2, e1600723. [CrossRef] [PubMed]

90. Ackermann, H.; Hage, S.R.; Ziegler, W. Brain Mechanisms of Acoustic Communication in Humans and Nonhuman Primates: An Evolutionary Perspective. Behav. Brain Sci. 2014, 37, 529-546. [CrossRef] [PubMed]

91. Owren, M.J.; Amoss, R.T.; Rendall, D. Two Organizing Principles of Vocal Production: Implications for Nonhuman and Human Primates. Am. J. Primatol. 2011, 73, 530-544. [CrossRef] [PubMed]

92. Scherer, K.R.; Johnstone, T.; Klasmeyer, G. Vocal Expression of Emotion. In Handbook of Affective Sciences; Series in affective science; Oxford University Press: New York, NY, USA, 2003; pp. 433-456.

93. Snowdon, C.T.; Teie, D. Affective Responses in Tamarins Elicited by Species-Specific Music. Biol. Lett. 2010, 6, 30-32. [CrossRef] [PubMed]

94. Lewis, P.A.; Critchley, H.D.; Rotshtein, P.; Dolan, R.J. Neural Correlates of Processing Valence and Arousal in Affective Words. Cereb. cortex 2007, 17, 742-748. [CrossRef] [PubMed]

95. Altenmüller, E.; Schmidt, S.; Zimmermann, E. Evolution of Emotional Communication: From Sounds in Nonhuman Mammals to Speech and Music in Man; Altenmüller, E., Schmidt, S., Zimmermann, E., Eds.; Oxford University Press: Oxford, UK, 2013.

96. Roberts, G.; Lewandowski, J.; Galantucci, B. How Communication Changes When We Cannot Mime the World: Experimental Evidence for the Effect of Iconicity on Combinatoriality. Cognition 2015, 141, 52-66. [CrossRef] [PubMed]

97. Monaghan, P.; Shillcock, R.C.; Christiansen, M.H.; Kirby, S. How Arbitrary Is Language? Philos. Trans. R. Soc. Lond. B Biol. Sci. 2014, 369, 20130299. [CrossRef] [PubMed]

98. Aryani, A. Affective Iconicity in Language and Poetry, Freie Universität Berlin. Ph.D. Dissertation, 2018. Available online: https:/ / refubium.fu-berlin.de/handle/fub188/22744 (accessed on 1 March 2019).

99. Aryani, A.; Conrad, M.; Schmidtke, D.; Jacobs, A. Why "piss" Is Ruder than "Pee"? The Role of Sound in Affective Meaning Making. PLoS ONE 2018, 13, e0198430. [CrossRef] [PubMed]

100. Aryani, A.; Hsu, C.-T.; Jacobs, A.M. The Sound of Words Evokes Affective Brain Responses. Brain Sci. 2018, 8, 94. [CrossRef] [PubMed]

101. Ullrich, S.; Kotz, S.A.; Schmidtke, D.S.; Aryani, A.; Conrad, M. Phonological Iconicity Electrifies: An ERP Study on Affective Sound-to-Meaning Correspondences in German. Front. Psychol. 2016, 7, 1200. [CrossRef] [PubMed]

102. Aryani, A.; Jacobs, A.M. Affective Congruence between Sound and Meaning of Words Facilitates Semantic Decision. Behav. Sci. 2018, 8, 56. [CrossRef] [PubMed]

103. Heffner, H.E.; Heffner, R.S. The Evolution of Mammalian Sound Localization. Acoust. Today 2016, $12,20-27$. 
104. Rendall, D.; Notman, H.; Owren, M.J. Asymmetries in the Individual Distinctiveness and Maternal Recognition of Infant Contact Calls and Distress Screams in Baboons. J. Acoust. Soc. Am. 2009, 125, 1792-1805. [CrossRef] [PubMed]

105. Monrad-Krohn, G.H. The Third Element of Speech: Prosody and Its Disorders. In Problems in Dynamic Neurology; Halpern, L., Ed.; Hebrew University Press: Jerusalem, Israel, 1963; pp. 101-117.

106. Belin, P.; Fillion-Bilodeau, S.; Gosselin, F. The Montreal Affective Voices: A Validated Set of Nonverbal Affect Bursts for Research on Auditory Affective Processing. Behav. Res. Methods 2008, 40, 531-539. [CrossRef] [PubMed]

107. Scherer, K.R. Expression of Emotion in Voice and Music. J. Voice 1995, 9, 235-248. [CrossRef]

108. Schröder, M. Experimental Study of Affect Bursts. Speech Commun. 2003, 40, 99-116. [CrossRef]

109. Paquette, S.; Peretz, I.; Belin, P. The "Musical Emotional Bursts": A Validated Set of Musical Affect Bursts to Investigate Auditory Affective Processing. Front. Psychol. 2013, 4, 509. [CrossRef] [PubMed]

110. Ekman, P.; Friesen, W.V.; Hager, J.C.; A Human Face (Firm). Facial Action Coding System; A Human Face: Salt Lake City, UT, USA, 2002.

111. Hauser, M.D. The Sound and the Fury: Primate Vocalizations as Reflections of Emotion and Thought. In The Origins of Music; Wallin, N.L., Brown, S., Merker, B., Eds.; The MIT Press: Cambridge, MA, USA, 2000; pp. 77-102.

112. Bryant, G.A. Animal Signals and Emotion in Music: Coordinating Affect across Groups. Front. Psychol. 2013, 4, 1-13. [CrossRef] [PubMed]

113. Seifert, U.; Verschure, P.F.M.J.; Arbib, M.A.; Cohen, A.J.; Fogassi, L.; Fritz, T.; Kuperberg, G.; Manzolli, J.; Rickard, N. Semantics of Internal and External Worlds. In Language, Music, and the Brain; Arbib, M.A., Ed.; The MIT Press: Cambridge, MA, USA, 2013; pp. 203-230.

114. Newman, J.D. Neural Circuits Underlying Crying and Cry Responding in Mammals. Behav. Brain Res. 2007, 182, 155-165. [CrossRef] [PubMed]

115. Filippi, P.; Congdon, J.V.; Hoang, J.; Bowling, D.L.; Reber, S.A.; Pašukonis, A.; Hoeschele, M.; Ocklenburg, S.; De Boer, B.; Sturdy, C.B.; et al. Humans Recognize Emotional Arousal in Vocalizations across All Classes of Terrestrial Vertebrates: Evidence for Acoustic Universals. Proc. R. Soc. B Biol. Sci. 2017, 284, 20170990. [CrossRef] [PubMed]

116. Köhler, W. Gestalt Psychology; An Introduction to New Concepts in Modern Psychology, Rev. ed.; Liveright: Oxford, UK, 1947.

117. Fort, M.; Martin, A.; Peperkamp, S. Consonants Are More Important than Vowels in the Bouba-Kiki Effect. Lang. Speech 2015, 58, 247-266. [CrossRef] [PubMed]

118. Morton, E.S. On the Occurrence and Significance of Motivation-Structural Rules in Some Bird and Mammal Sounds. Am. Nat. 1977, 111, 855-869. [CrossRef]

119. Ohala, J. Signaling with the Eyebrows-Commentary on Huron, Dahl, and Johnson. Empir. Musicol. Rev. 2009, 4, 101-102. [CrossRef]

120. Sievers, B.; Polansky, L.; Casey, M.; Wheatley, T. Music and Movement Share a Dynamic Structure That Supports Universal Expressions of Emotion. Proc. Natl. Acad. Sci. USA 2013, 110, 70-75. [CrossRef] [PubMed]

121. Trevarthen, C. Musicality and the Intrinsic Motive Pulse: Evidence from Human Psychobiology and Infant Communication. Music. Sci. 1999, 3, 155-215. [CrossRef]

122. Johnstone, T.; Scherer, K.R. Vocal Communication of Emotion. In Handbook of Emotions; Lewis, M., Haviland-Jones, J.M., Eds.; The Guilford Press: New York, NY, USA, 2000.

123. Massaro, D.W. Multimodal Emotion Perception: Analogous to Speech Processes. In Proceedings of the ISCA Workshop on Speech and Emotion, Newcastle, Northern Ireland, UK, 5-7 September 2000; pp. 114-121.

124. Gallese, V.; Fadiga, L.; Fogassi, L.; Rizzolatti, G. Action Recognition in the Premotor Cortex. Brain 1996, 119, 593-609. [CrossRef] [PubMed]

125. McGurk, H.; MacDonald, J. Hearing Lips and Seeing Voices. Nature 1976, 264, 746-748. [CrossRef] [PubMed]

126. Merker, B. Synchronous Chorusing and Human Origins. In The Origins of Music; Wallin, N.L., Merker, B., Brown, S., Eds.; The MIT Press: Cambridge, MA, USA, 2000; pp. 315-327.

127. Stainsby, T.; Cross, I. The Perception of Pitch. In The Oxford Handbook of Music Psychology; Hallam, S., Cross, I., Thaut, M., Eds.; Oxford University Press: Oxford, NY, USA, 2008; Volume 1, pp. 47-58. 
128. Peng, S.-C.; Chatterjee, M.; Lu, N. Acoustic Cue Integration in Speech Intonation Recognition With Cochlear Implants. Trends Amplif. 2012, 16, 67-82. [CrossRef] [PubMed]

129. Banse, R.; Scherer, K.R. Acoustic Profiles in Vocal Emotion Expression. J. Personal. Soc. Psychol. 1996, 614-636. [CrossRef]

130. Bradley, M.M.; Lang, P.J. Affective Reactions to Acoustic Stimuli. Psychophysiology 2000, 37, $204-215$. [CrossRef] [PubMed]

131. Xu, L.; Thompson, C.S.; Pfingst, B.E. Relative Contributions of Spectral and Temporal Cues for Phoneme Recognition. J. Acoust. Soc. Am. 2005, 117, 3255-3267. [CrossRef] [PubMed]

132. McAdams, S.; Giordano, B.L. The Perception of Musical Timbre. In The Oxford Handbook of Music Psychology; Hallam, S., Cross, I., Thaut, M., Eds.; Oxford University Press: Oxford, NY, USA, 2008; Volume 1, pp. 72-80. [CrossRef]

133. Fu, Q.-J.; Zeng, F.-G.; Shannon, R.V.; Soli, S.D. Importance of Tonal Envelope Cues in Chinese Speech Recognition. J. Acoust. Soc. Am. 1998, 104, 505-510. [CrossRef] [PubMed]

134. Lehiste, I. The Function of Quantity in Finnish and Estonian. Language 1965, 41, 447-456. [CrossRef]

135. Suomi, K. Temporal Conspiracies for a Tonal End: Segmental Durations and Accentual F0 Movement in a Quantity Language. J. Phon. 2005, 33, 291-309. [CrossRef]

136. Järvikivi, J.; Vainio, M.; Aalto, D. Real-Time Correlates of Phonological Quantity Reveal Unity of Tonal and Non-Tonal Languages. PLoS ONE 2010, 5, e12603. [CrossRef] [PubMed]

(C) 2019 by the authors. Licensee MDPI, Basel, Switzerland. This article is an open access article distributed under the terms and conditions of the Creative Commons Attribution (CC BY) license (http://creativecommons.org/licenses/by/4.0/). 